Proceedings the 44th International School and Conference on the Physics of Semiconductors "Jaszowiec 2015", Wisła 2015

\title{
Spin Conductance of Nanowires with Double Coupled Quantum Dots
}

\author{
M. WoŁoszyn, B.J. Spisak, P. Wójcik and J. Adamowski* \\ AGH University of Science and Technology, Faculty of Physics and Applied Computer Science, \\ al. Mickiewicza 30, 30-059 Kraków, Poland
}

\begin{abstract}
Using the computer simulations we have studied the spin conductance of the InAs nanowire with three allaround gates that generate two coupled quantum dots in the nanowire. We have assumed that the same constant voltage is applied to the outermost (left and right) gates and investigated the effect of the varying central-gate voltage $\left(V_{\mathrm{G}}\right)$ and axially directed magnetic field $B$ on the spin currents. The calculated spin-up and spin-down conductances exhibit pronounced oscillations as functions of $V_{\mathrm{G}}$. In certain intervals of $V_{\mathrm{G}}$, both the spin conductances oscillate in antiphase, which can be applied to the spin-filter operation.
\end{abstract}

DOI: 10.12693/APhysPolA.129.A-114

PACS: 72.25.Dc, 73.63.Nm

\section{Introduction}

The gates attached to the semiconductor nanowire can generate the electrostatic (gate-controlled) quantum dots (QDs) in the nanostructure. The gates can be fabricated in either the planar [1, 2] or ring [3] geometry, so-called gate-all-around (GAA) [4]. Pfund et al. [1] performed transport measurements on double QDs created by top gates in InAs nanowire and demonstrated the full tunability from strong to weak coupling between the molecular states formed in QDs. Fuhrer et al. [2] studied the single-electron pumping in double QDs electrically defined in InAs nanowire and demonstrated the suitability of coupled QDs in nanowires for electron pumping applications. Larrieu and Han [4] investigated the vertical nanowire-based field-effect transistors and showed that the transistor architecture based on the parallel GAA nanowires leads to high electrical performance. Gúl et al. [3] performed magnetotransport measurements on GaAs/InAs core-shell nanowires with the GAA and found periodic oscillations of the magnetoconductance.

In the present work, we calculated spin currents in the InAs GAA nanowire with the two coupled QDs in the axially oriented magnetic field and demonstrated that the spin conductances can be tuned by the gate voltage.

\section{Theoretical model and computational method}

We consider the InAs nanowire with three ring gates (Fig. 1a). If all the gates are negatively polarized, potential $V$ in the nanowire possesses the profile shown in Fig. 1b. Accordingly, the electron potential energy $U=-e V$, where $e>0$ is the elementary charge, exhibits the triple-barrier shape with the two potential wells. This means that the two coupled QDs are formed in the nanowire. In the present study, we assume that

*corresponding author; e-mail: adamowski@fis.agh.edu.pl the constant voltage $V_{\mathrm{L}}=V_{\mathrm{R}}$ is applied to the outermost (left and right) gates, while the voltage $V_{\mathrm{G}}$ applied to the central gate allows us to tune the coupling between the QDs.

(a)
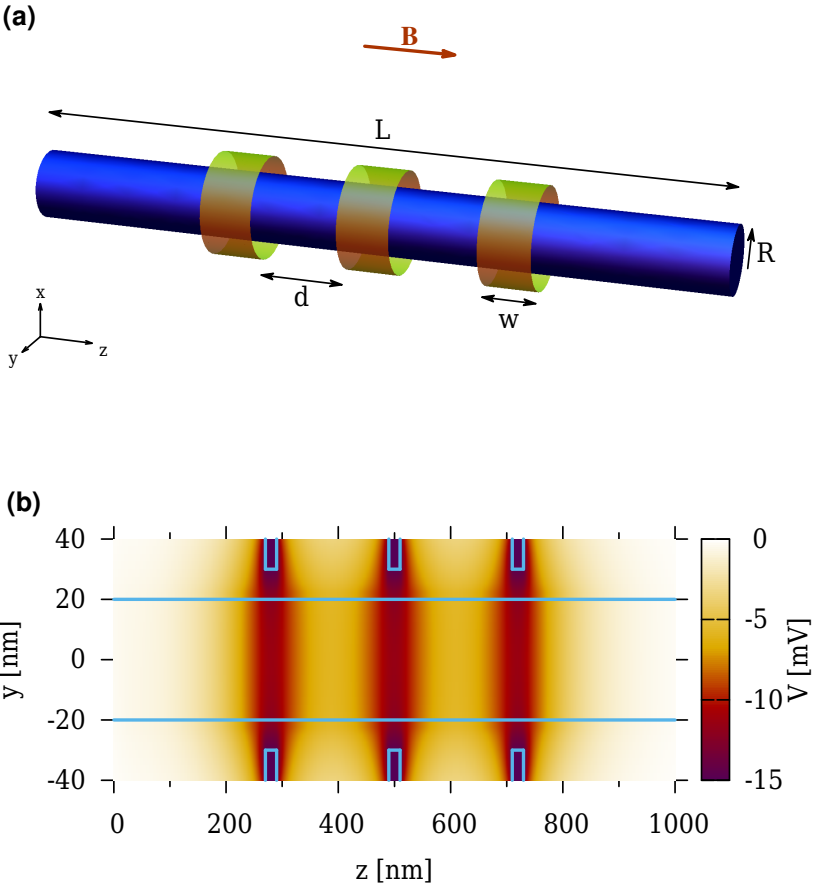

Fig. 1. (a) Schematic of the nanowire with three ring (GAA) gates. (b) Cross-section $(x=0)$ of the potential profile in the case of equal gate potentials, $V_{\mathrm{L}}=V_{\mathrm{G}}=$ $V_{\mathrm{R}}=-15 \mathrm{mV}$. Solid lines show the boundaries of the nanowire and the gates.

In the first step of calculations, we have numerically solved the three-dimensional (3D) Laplace equation in Cartesian coordinates (with $z$ axis directed along the 
nanowire axis). The calculated potential profile is depicted in Fig. 1b. In the second step, the one-electron wave functions and the associated energy levels have been calculated by the variational method with the 3D Gaussian basis functions [5]. The 3D potential energy in the nanowire can be approximately separated into the longitudinal ( $z$-dependent) and transverse $(x, y$-dependent) terms, which allows us to apply the adiabatic approximation [15] and determine the effective $z$-dependent potential profile. In the calculations, we have included the static homogeneous magnetic field $\boldsymbol{B}=(0,0, B)$ oriented along the nanowire axis and the spin Zeeman splitting $\Delta E_{Z}=g^{\star} \mu_{\mathrm{B}} B$, where $g^{\star}$ is the effective Landé factor and $\mu_{\mathrm{B}}$ is the Bohr magneton. In the last step, we have calculated the spin-dependent transmission coefficients using the quantum transmitting boundary method [6] and the spin-dependent conductance with the help of the Landauer formula.

Due to the approximate separation of the potential energy in the nanowire into the longitudinal and transverse parts the one-electron energy levels can be written down as follows: $E_{m n}=E_{m}^{\|}+E_{n}^{\perp}$, where $E_{m}^{\|}$are the energies of the quasi-bound $z$-dependent states and $E_{n}^{\perp}$ are the energies of transverse modes that form the conduction channels. The spin Zeeman interaction causes that energy levels $E_{m}^{\|}$also depend on spin quantum numbers $\sigma=(\uparrow, \downarrow)$. We have set Fermi energy $E_{F}=30 \mathrm{meV}$ so that only the lowest-energy conduction channel $(n=0)$ with energy $E_{0}^{\perp}=19.1 \mathrm{meV}$ for $B=0$ is activated in the transport.

The present calculations have been performed with the following material parameters: nanowire length $L=$ $1000 \mathrm{~nm}$, nanowire radius $R=20 \mathrm{~nm}$, each GAA has internal radius $30 \mathrm{~nm}$ and width $w=20 \mathrm{~nm}$, and is separated from the nanowire by the $10 \mathrm{~nm}$ thick insulator $\left(\mathrm{SiO}_{2}\right)$, distance $d$ between the adjacent gates is $200 \mathrm{~nm}$ (Fig. 1a). The constant voltage $\left(V_{\mathrm{L}}=V_{\mathrm{R}}=-15 \mathrm{mV}\right)$ is applied to the left and right gates, while voltage $V_{\mathrm{G}}$ applied to the central gate is changed from $-20 \mathrm{mV}$ to 0 . Moreover, we take on the InAs band mass $m_{e}=$ $0.0265 m_{e 0}$, where $m_{e 0}$ is the electron rest mass, effective Landé factor $g^{\star}=-15.0$, and electric permittivities 15.1 for InAs and 3.9 for $\mathrm{SiO}_{2}$.

\section{Results and discussion}

The conductance $G_{\sigma}$ calculated for spin-up $(\sigma=\uparrow)$ and spin-down $(\sigma=\downarrow)$ electrons is depicted in Fig. 2. We observe the oscillations of the conductance as a function of central-gate voltage $V_{\mathrm{G}}$. Each peak of the conductance results from the resonant tunneling that occurs if energy level $E_{m}^{\|}$aligns with Fermi energy $E_{F}$. Energy levels $E_{m}^{\|}$are changed by changing the gate voltage $V_{\mathrm{G}}$, i.e., if we increase the (negative) gate voltage, the central potential energy barrier is lowered, which leads to the lowering of the energy levels that subsequently cross the Fermi level. In certain intervals of the gate voltage, e.g., $-14 \mathrm{mV} \leq V_{\mathrm{G}} \leq 0$ for $B=0.4 \mathrm{~T}$, both the spin-dependent conductances oscillate in antiphase. This means that the GAA nanowire shows the spin filtering effect.

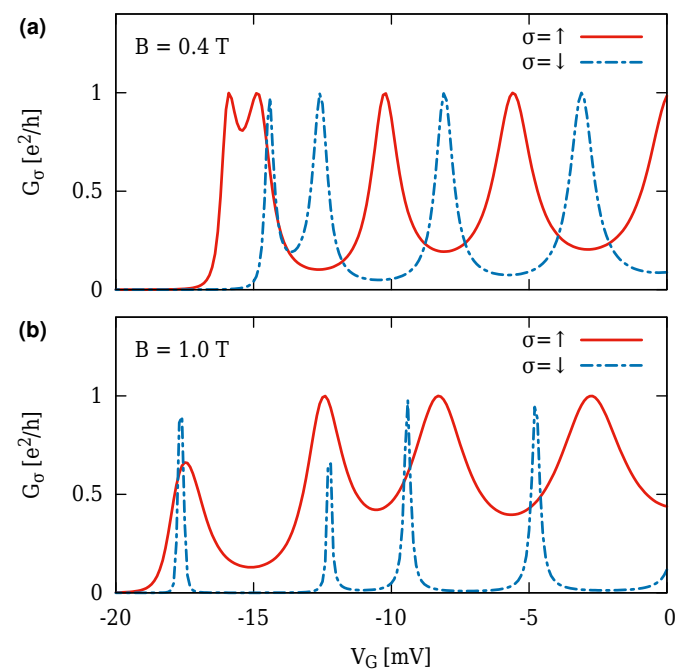

Fig. 2. Conductance $G_{\sigma}$ for spin up $(\sigma=\uparrow)$ and spin down $(\sigma=\downarrow)$ electrons as a function of central-gate voltage $V_{\mathrm{G}}$ for magnetic field (a) $B=0.4 \mathrm{~T}$ and (b) $B=1.0 \mathrm{~T}$.

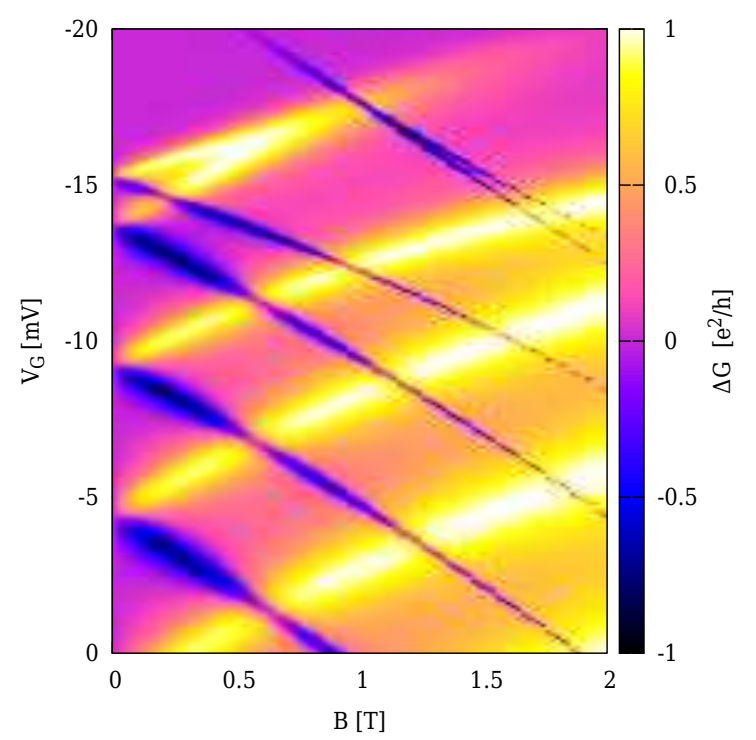

Fig. 3. Spin conductance $\Delta G=G_{\uparrow}-G_{\downarrow}$ of the nanowire with three ring gates as a function of centralgate voltage $V_{\mathrm{G}}$ and magnetic field $B$.

In Fig. 3, the spin conductance $\Delta G=G_{\uparrow}-G_{\downarrow}$ is displayed on the $B-V_{\mathrm{G}}$ plane. The dark (light) narrow bands correspond to the spin currents with the majority spins down (up). The increasing magnetic field and decreasing central-gate voltage causes that the transitions between the regimes with the spin polarized current and unpolarized current become more pronounced ( $c f$. upper left part of Fig. 3). 


\section{Conclusions}

We have shown how the spin currents can be generated and modified in the GAA nanowire with three gates, i.e., two coupled QDs, in the magnetic field. The present results demonstrate the good tunability of the nanodevice based on the coupled QDs, which results from the field effect of the central ring gate. The GAA nanowire studied in the present paper can operate as the spin filter controlled by the gates.

\section{Acknowledgments}

This project is supported by the National Science Centre, Poland, under grant DEC-2011/03/B/ST3/00240.

\section{References}

[1] A. Pfund, I. Shorubalko, R. Leturcq, K. Ensslin, Appl. Phys. Lett. 89, 252106 (2006).

[2] A. Fuhrer, C. Fasth, L. Samuelson, Appl. Phys. Lett. 91, 052109 (2007).

[3] Ö. Gül, N. Demarina, C. Blömers, T. Rieger, H. Lüth, M.I. Lepsa, D. Grützmacher, Th. Schäpers, Phys. Rev. B 89, 045417 (2014).

[4] G. Larrieu, X.-L. Han, Nanoscale 5, 2437 (2013).

[5] M. Wołoszyn, B.J. Spisak, J. Adamowski, P. Wójcik, J. Phys. Condens. Matter 26, 325301 (2014).

[6] C.S. Lent, D.J. Kirkner, J. Appl. Phys. 67, 6353 (1990). 